\title{
招請講演
}

\section{Advances in Pediatric Urology Which Have Affected the Management of Bladder Exstrophy}

\author{
The Johns Hopkins Hospital \\ Robert D. Jeffs
}

The pediatric urology from tentative beginnings has grown into a significant subspecialty. Advances seen in pediatric urology have been applied to many urological problems of children. These advances have had a particular impact on the management of the exstrophy epispadias complex. Classical exstrophy patients treated 50 years ago frequently had a poor result in regard to continence, renal preservation, cosmetic reconstruction and sexuality. Functional reconstruction in exstrophy patients entirely managed in our institution has improved to a point where we expect to obtain continence in greater than $80 \%$ of them. Advances in anesthesia, pain control, fluid and electrolyte management have allowed long and difficult procedures to be performed in infants and young children. Infection control by antibiotics has made it possible to close contaminated bladders with primary healing of the suture lines. The imperfections of drainage and reflux can be managed by antibiotic suppression until definitive repair is complete.

Advances in bladder physiology and urodynamics have allowed a better understanding of the potential of the exstrophy bladder. The low outlet resistance of the initial bladder closure and the subsequent epispadias repair provide low levels of tension in the biadder wall which stimulate growth and increase capacity. The bladder must demonstrate its ability to adapt to increasing volume before the operation for continence is attempted.

The bladder neck reconstruction in adapted form Guy Leadbetter's tubularization of the trigone to produce resistance and is enhanced by the suspension of the bladder neck in the Marshall Marchetti manner. Ureteral reimplantation is part of the bladder neck procedure and represents one of the great advances in pediatric urology. Control of renal infection by proper understanding and management of reflux is cantral to so many problems in pediatric urology and its management is key to success in staged reconstruction of the exstrophy patient.

The success of closure and the eventual continence are aided by closure of the pelvic ring and muscles of the pelvic floor. A variety of types of pelvic osteotomy have been suggested but anterior innominate osteotomy and its modifications is favoured.

Evaluation, planning and repair of the epispadias condition of the external genitalia in both male and female patients is an important part of reconstuction. Penile length, chordee, erection and ejaculation must be considered at each stage of reconstruction. The open urethra is not divided at the time of initial closure to avoid strictures. Growth and length usually is sufficient by the time epispadias repair is made in the Cantwell Ransley manner. In the male, testosterone stimulation has been found helpful in preparation for epispadias repair. In rare instances at birth when the phallus is short, reassignment of the sex of rearing from male to female can be considered.

Reconstruction in the exstrophy patient is not always possible and when attempted is not always successful. The many innovations applied to pediatric urology have made it possible to provide acceptable alternatives to normal bladder function for these patients. Clean intermittent catheterization, augmentation, abdominal stomata and internal 
diversion provide acceptable alternatives for those who can be reconstructed.

Evaluation of the psychosocial development and eventual fertility is part of the long-term management. Furhter advances and adaptation of new tech niques which can be applied to the exstrophy prob- lem will undoubtedly occur. Prenatal diagnosis is unlikely to remove the problem from pediatric urology entirely and consequently new advances in this specialty will continue to be applied to this difficult condition. 